\title{
UNA NUEVA ESPECIE DE CATOPSIS (BROMELIACEAE, TILLANDSIOIDEAE, CATOPSIDEAE) DE MÉXICO
}

\author{
Nancy Martínez-Correa ${ }^{1,3}$, Adolfo Espejo-Serna ${ }^{2}$ y Ana Rosa López-Ferrari ${ }^{2}$ \\ ${ }^{1}$ Universidad Autónoma Metropolitana, División de Ciencias Biológicas y de la \\ Salud, Programa de Doctorado en Ciencias Biológicas y de la Salud, \\ México, D.F., México. \\ ${ }^{2}$ Universidad Autónoma Metropolitana-Iztapalapa, División de \\ Ciencias Biológicas y de la Salud, Departamento de Biología, Herbario \\ Metropolitano "Ramón Riba y Nava Esparza", \\ Apdo. postal 55-535, 09340 México, D.F., México. \\ ${ }^{3}$ Autor para la correspondencia: correanm@yahoo.com.mx
}

\section{RESUMEN}

Se describe e ilustra Catopsis occulta Mart.-Correa, Espejo \& López-Ferr., especie nueva endémica de los estados de Chiapas, Oaxaca y Veracruz en México. El nuevo taxon se caracteriza por sus plantas dioicas de gran tamaño con las inflorescencias generalmente tres veces ramificadas y las brácteas florales y los sépalos amarillo-anaranjados. Se le compara con C. berteroniana (Schult. \& Schult. f.) Mez, la cual presenta características morfológicas similares.

Palabras clave: Bromeliaceae, Catopsis, dioicismo, endemismo.

\begin{abstract}
Catopsis occulta Mart.-Correa, Espejo \& López-Ferr., a new endemic species from the states of Chiapas, Oaxaca and Veracruz in Mexico, is described and illustrated. The new taxon is characterized by dioecious, large plants with usually three times branched inflorescences and yellow-orange floral bracts and sepals. The new species is compared with C. berteroniana (Schult. \& Schult. f.) Mez, which is morphologically similar.
\end{abstract}

Key words: Bromeliaceae, Catopsis, dioecious, endemism. 
El género Catopsis fue descrito por Grisebach (1864), quien lo ubicó en la tribu Tillandsieae de la subfamilia Tillandsioideae junto con otros géneros que actualmente están incorporados en las subfamilias Tillandsioideae y Pitcairnioideae. Las plantas de Catopsis se distinguen por el siguiente conjunto de caracteres: presencia del apéndice seminal, el cual se origina de proyecciones epidérmicas de la calaza para formar un coma apical enrollado, el margen entero y simple de la apertura del grano de polen, la presencia de ceras epicuticulares blancas en la gran mayoría de las especies y los sépalos fuertemente asimétricos (Smith y Downs, 1977; Palací, 1997; Smith y Till, 1998; Barfuss et al., 2005). Otra peculiar característica del género es el dioicismo, presente en $61 \%$ de sus especies, condición conocida únicamente en cinco géneros de la familia, de los cuales dos pertenecen a Bromelioideae (Aechmea y Androlepis), uno a Pitcairnioideae (Dyckia), uno a Hechtioideae (Hechtia) y uno a Tillandsioideae (Catopsis) (Smith y Downs, 1974; 1977; 1979; Burt-Utley y Utley, 1988; Robinson y Taylor, 1999). Todas las características arriba mencionadas, diferencian a Catopsis del resto de los géneros de la subfamilia Tillandsioideae, y por ello algunos autores han considerado colocarlo en una tribu propia: Catopsideae (Harms, 1930; Barfuss et al., 2005).

Incluyendo al nuevo taxon aquí propuesto, Catopsis comprende 21 especies (Smith y Downs, 1977; Palací, 1997; Espejo-Serna et al., 2004; Barfuss et al., 2005; Luther, 2012), de las cuales 18 son epífitas y tres (C.floribunda (Brongn.) L. B. Sm., C. morreniana Mez y C. occulta Mart.-Correa, Espejo \& López-Ferr.) pueden ser tanto epífitas como rupícolas. El área de distribución de Catopsis comprende Estados Unidos (Florida), las Bahamas, México, Cuba, Haití, República Dominicana, Puerto Rico, Islas Vírgenes (británicas y estadounidenses), Islas de Barlovento, Jamaica, Guatemala, Belice, Honduras, El Salvador, Nicaragua, Costa Rica, Panamá, Colombia, Venezuela, República de Trinidad y Tobago, Guyana, Surinam, Guayana Francesa, Ecuador, Perú y Brasil (Urban, 1903-1911; Standley, 1928; Smith, 1938; Smith y Lundell, 1940; Carabia, 1941; Moscoso, 1943; Smith, 1943; Gilmartin, 1965; Proctor y Read, 1972; Liogier, 1974; Ariza-Julia y Liogier, 1977; Smith y Downs, 1977; Liogier y Martorell, 1982; Zanoni et al., 1986; Forero y Gentry, 1989; Luther, 1989; Gómez y Winkler, 1991; Holst, 1994; Huft, 1994; Luther, 1995; Boggan et al., 1997; Palací, 1997; Rivas et al., 1997; Vásquez, 1997; Krömer et al., 1999; ZavalaMolina, 2002; Espejo-Serna et al., 2004; Versieux et al., 2008).

De las 21 especies del género, 18 están presentes en México, solo Catopsis micrantha L. B. Sm., C. pisiformis Rauh y C. werckleana Mez no se conocen del país. C. compacta Mez, C. paniculata E. Morren, C. pendula Baker y C. occulta Mart.-Correa, Espejo \& López-Ferr., aquí propuesta, son, hasta donde sabemos, endémicas del territorio mexicano (Espejo Serna, 2012). 
Mez (1896) propuso, basándose en el sexo de las flores y en la longitud de los estambres, una clasificación infragenérica para Catopsis, dividiéndolo en dos subgéneros: Eucatopsis y Tridynandra. En el primero, incluyó a las especies con flores bisexuales y filamentos todos del mismo largo y en el segundo, a las que tienen flores unisexuales y tres estambres o estaminodios cortos y tres largos. Sin embargo, esta clasificación no ha sido reconocida en tratamientos taxonómicos posteriores (Smith \& Downs, 1977; Palací, 1977).

En su tesis doctoral, Palací (1997) con base en la morfología floral y el sexo de las especies reconoció tres grandes grupos en Catopsis: el primero constituido por especies monoicas monoclinas ("homoecious" sensu Palací, 1997) (C. juncifolia Mez \& Wercklé y C. nitida (Hook.) Griseb.); el segundo formado por taxa dioicos (C. delicatula L. B. Sm., C. hahnii Baker, C. micrantha L. B. Sm., C. paniculata E. Morren y C. subulata L. B. Sm.) y finalmente un tercero constituido por aquellas que son trioicas con flores bisexuales ("perfect” sensu Palací, 1997), pistiladas o estaminadas ("homoecious and dioecious populations" sensu Palací, 1997) (C. nutans (Sw.) Griseb., C. sessiliflora (Ruiz \& Pav.) Mez). En este último grupo existen, de acuerdo con Palací, dos subgrupos, uno con algunas especies predominantemente monoicas monoclinas ("homoecious" sensu Palací, 1997)) con poblaciones dioicas raras u ocasionales (C. berteroniana (Schult. \& Schult f.) Mez, C. floribunda (Brongn.) L. B. Sm. y C. wangerinii Mez \& Wercklé ex Mez) y otro con plantas predominantemente dioicas, con poblaciones monoicas monoclinas ("homoecious" senus Palací, 1997)) ocasionales (C. morreniana Mez).

Después de analizar 1867 especímenes herborizados (1741 números de colecta) y 802 disecciones florales, pertenecientes a las 21 especies de Catopsis y relacionarlos con sus patrones de distribución geográfica, concluimos que en el género existen taxa monoicos monoclinos, taxa dioicos y taxa trioicos. Entendiendo por monoico monoclino ("homoecious" sensu Palací, 1997) la condición de tener flores bisexuales, a diferencia de monoico diclino, que alude a la presencia de flores pistiladas y estaminadas en el mismo individuo. Las especies que presentan plantas pistiladas y plantas estaminadas son dioicas y finalmente el término trioico hace referencia a las especies con plantas pistiladas, plantas estaminadas y plantas monoicas monoclinas (Font Quer, 1977; Harris \& Wolf, 1997).

Toda esta complicada situación de la distribución de los sexos en las especies del género ha propiciado que su comprensión y delimitación taxonómica sean deficientes. Un ejemplo de lo antes mencionado es el caso de Catopsis berteroniana, descrita por J. A. Schultes y J. H. Schultes (1830, como Tillandsia berteroniana) a partir de un espécimen recolectado por Bertero en República Dominicana depositado en el 
herbario de Balbis (actualmente resguardado en la Universidad de Turín (TO) (Thiers, [continuosuly updated])). Sin embargo y de acuerdo con la dra. Laura Guglielmone, curadora de dicha colección, en el herbario Balbis, incorporado actualmente al acervo general, existe solo un ejemplar de T. berteroniana ( $=$ C. berteroniana) (Fig. 1), el cual no es el ejemplar tipo del nombre en cuestión. Aparentemente, dicho tipo se encuentra perdido y por tal motivo, con el propósito de contar con una referencia que regule la debida aplicación del nombre, hemos decidido designar un neotipo:

Tillandsia berteroniana Schult. \& Schult. f., Syst. Veg. 7: 1221-1222. 1830. Tipo: In St. Domingo: Bertero (TO?). Neotipo, aquí designado: República Dominicana, cordillera central, provincia Dajabón, $32 \mathrm{~km}$ sur de Loma de Cabrera (5.7 $\mathrm{km}$ norte de Villa Anacaona) en la carretera internacional, bosque seco de Pinus occidentalis con un bosque denso de Syzygium jambos en el arroyo del río, $19^{\circ} 16^{\prime}$ N, 71²47 W, 1900 ft, 5 marzo 1982, T. Zanoni, M. Mejía, J. Pimentel \& J. T. Mickel 19658 (Neotipo: JBSD, Isoneotipo: MO) (Figs. 2 y 6).

Como parte de la revisión sistemática del género que estamos realizando, la cual ha incluido recolecciones y observaciones de plantas vivas, así como revisión de material herborizado procedente tanto de México como de Centroamérica y del Caribe, hemos concluido que el material identificado hasta ahora como Catopsis berteroniana (Schult. \& Schult. f.) Mez corresponde en realidad a dos especies diferentes, una monoica monoclina: C. berteroniana y otra dioica, que aquí proponemos como nueva para la ciencia:

Catopsis occulta Mart.-Correa, Espejo \& López-Ferr. sp. nov. Figs. 3A-D, 4A-B, 5A-B, D-E y 6.

Epiphyte, rarely saxicolous, rosulate herbs, dioecious, flowering up to 1.5 $\mathrm{m}$ high. Stem inconspicuous, short. Leaves numerous; sheaths 13-18(-23) cm long; blades light green, triangular to narrowly triangular, $13-25(-36) \mathrm{cm}$ long, glabrous. Inflorescence terminal, erect, three times branched, rarely two times branched; staminate inflorescence (9-)14-30 cm high; flowers sessile; sepals yellow-orange, oblong, 7.6-11 mm long, 3.9-6.3 mm wide; petals white, lanceolate, 6.2-10 mm long, 2-4.5 mm wide; stamens in two series of different length; filaments white, cylindrical, the longest 6-6.8 mm long, the shortest 3.7-5.4 mm long; anthers yellow, triangular, basifixed; pistillate inflorescence $20-50 \mathrm{~cm}$ high; flowers sessile; sepals yellow, oblong, 1.2-1.4 cm long, 6.4-10 mm wide; petals white, lanceolate-triangular, 1-1.5 $\mathrm{cm}$ long, 4.2-6.5 mm wide; ovary green, 1-1.2 cm long. Capsule brown, ovoid, 1.2$1.8 \mathrm{~cm}$ long; seeds fusiform, $1.5-2 \mathrm{~mm}$ long. 
Martínez-Correa et al.: Nueva especie de Catopsis (Bromeliaceae) de México

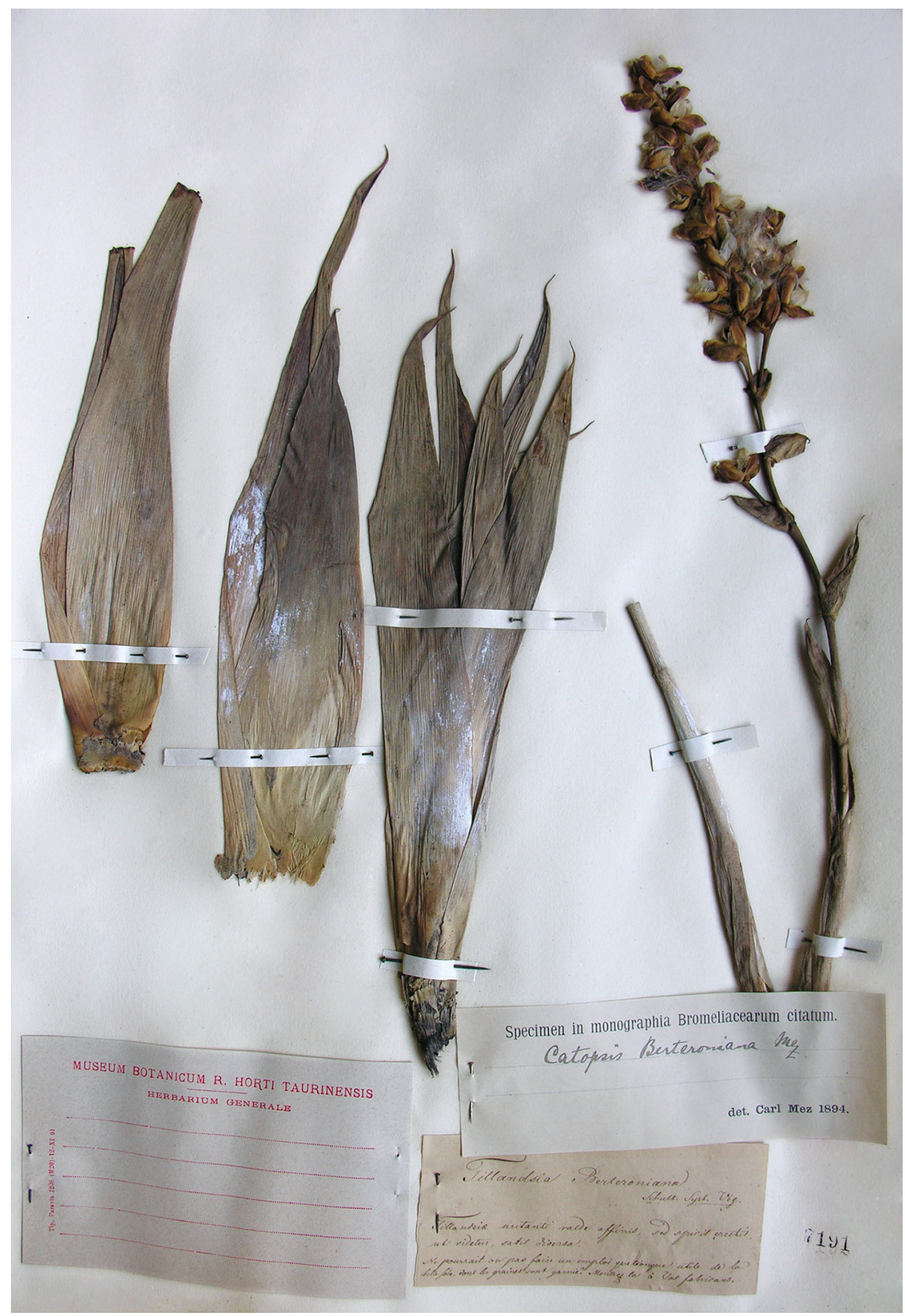

Fig. 1. Espécimen de Tillandsia berteroniana Schult. \& Schult. f. (= Catopsis berteroniana (Schult. \& Schult. f.) Mez) depositado en el herbario de la Universidad de Turín (TO). 

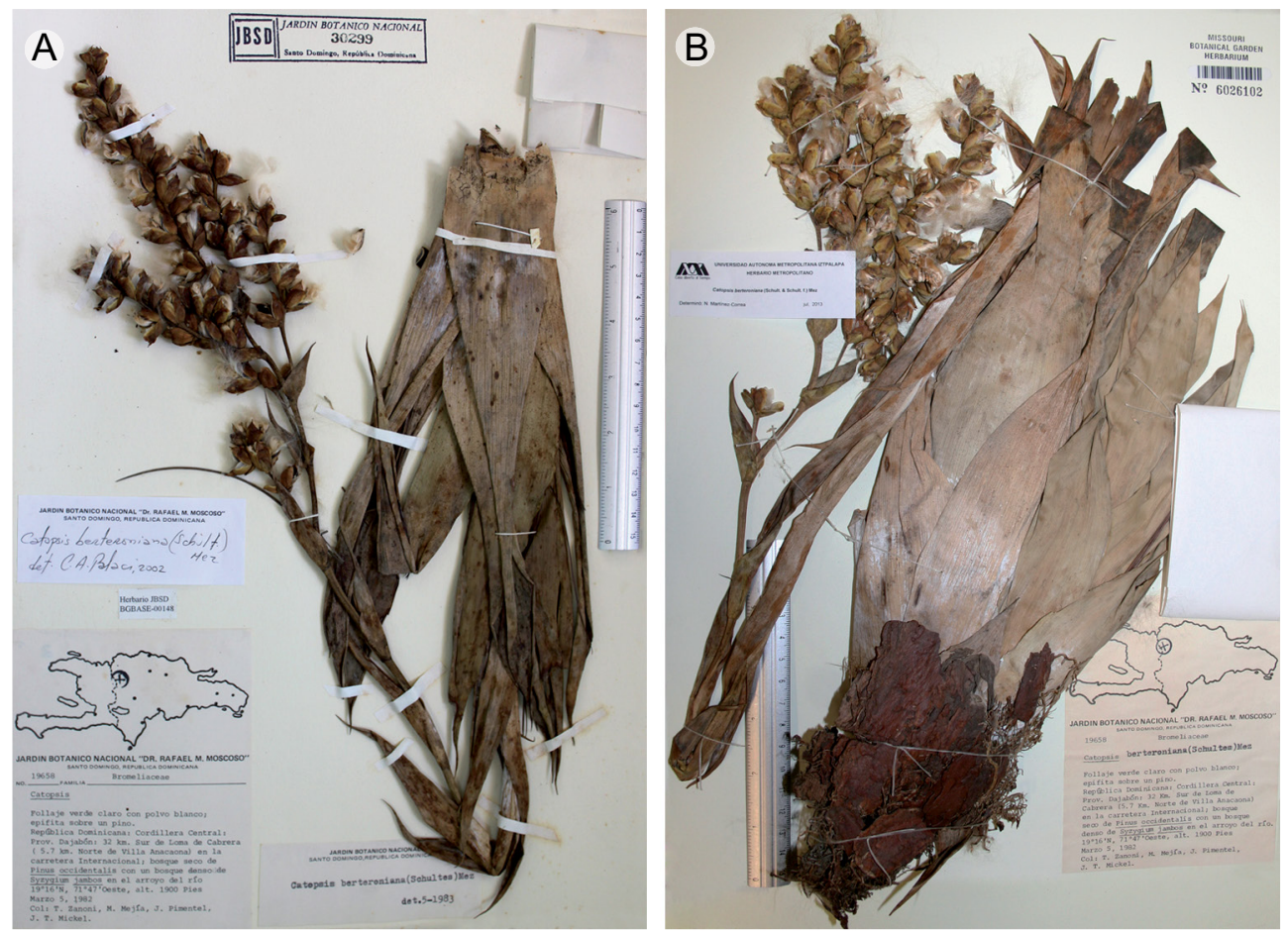

Fig. 2. Neotipo de Tillandsia berteroniana Schult. \& Schult. f. (= Catopsis berteroniana (Schult. \& Schult. f.) Mez): A. espécimen en el Jardín Botánico de Santo Domingo (JBSD); B. espécimen en el Jardín Botánico de Missouri (MO).

Hierbas perennes, dioicas, arrosetadas, acaules, pruinosas, en flor de hasta 1.5 $\mathrm{m}$ de alto, epífitas o más raramente rupícolas; rosetas de $(20-) 35-50 \mathrm{~cm}$ de alto por 20-30 cm de diámetro. Hojas numerosas, las vainas verdes claras, ovadas a cuadradas, de 13-18(-23) cm de largo, de 4-9(-11) cm de ancho, glabras; las láminas verdes claras, triangulares a largamente triangulares, de 13-25(-36) $\mathrm{cm}$ de largo, de 3-7 $\mathrm{cm}$ de ancho, glabras, el ápice atenuado. Inflorescencia terminal, erecta, tres o raramente dos veces dividida; la masculina de (9)14-30 cm de alto, con 5-10 ramas primarias; pedúnculo verde, cilíndrico, de 33-60 cm de largo, de 2.6-7.6 mm de diámetro; brácteas inferiores del pedúnculo lanceoladas, de 13-21 cm de largo, el ápice atenuado, mucho más largas que los entrenudos, brácteas superiores del pedúnculo lanceoladas, de 7.4-15 cm de largo, el ápice apiculado, más largas que los entrenudos; brácteas primarias inferiores lanceoladas, las superiores ovadas, de 2.7-6 cm de largo, de 1-2 cm de ancho, el ápice apiculado, más largas que el pedículo primario; brácteas secundarias, cuando pre- 


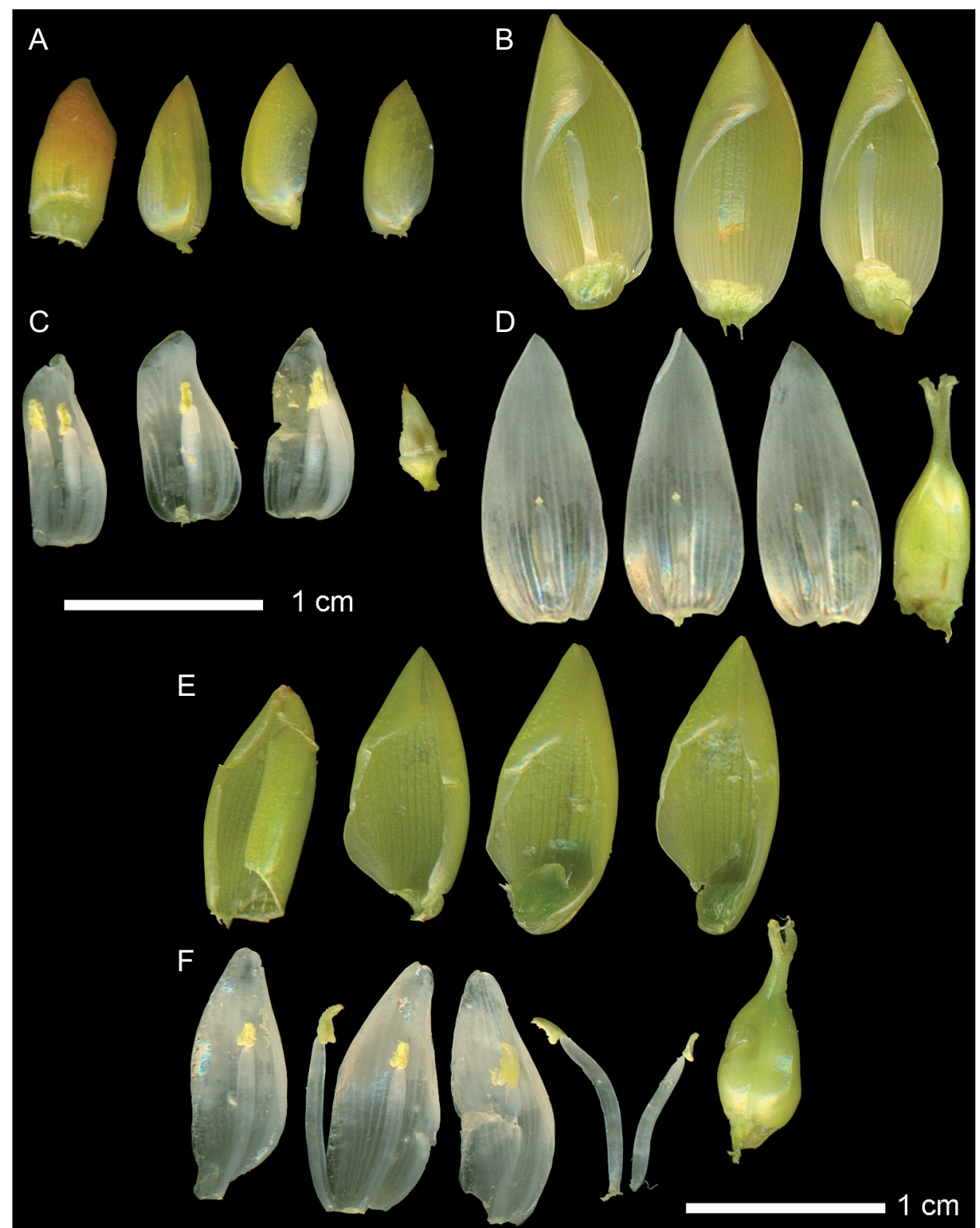

Fig. 3. Disecciones florales. Catopsis occulta Mart-Correa, Espejo \& López-Ferr. A. flor masculina, bráctea floral (izquierda) y sépalos (3, derecha); B. sépalos (3) y estaminodios, flor femenina; C. pétalos, estambres y pistilodio, flor masculina; D. pétalos, estaminodios y pistilo, flor femenina. Catopsis berteroniana (Schult. \& Schult. f.) Mez: E. bráctea floral y sépalos; F. pétalos, estambres y pistilo. 


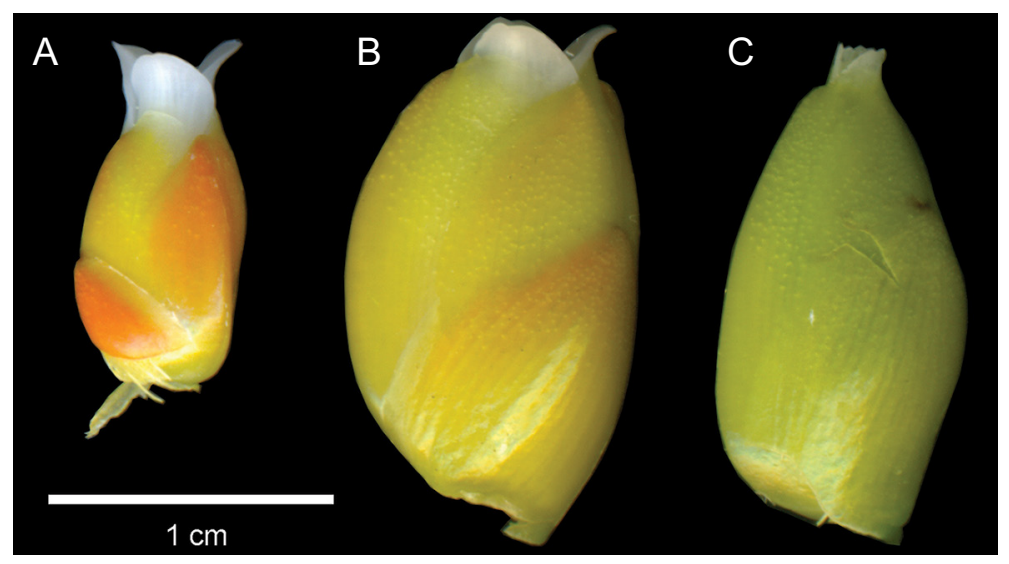

Fig. 4. Catopsis occulta Mart.-Correa, Espejo \& López-Ferr. A. flor masculina; B. flor femenina. Catopsis berteroniana (Schult. \& Schult. f.) Mez, C. flor bisexual.

sentes, ovado-lanceoladas, de 5.3-8.3 mm de largo, de 3.4-5.3 $\mathrm{mm}$ de ancho, el ápice agudo, más cortas que el pedículo secundario; espigas 8-19, de 5.3-11 cm de largo, de 0.7-1.3 cm de diámetro, con 19-30(-40) flores dispuestas helicoidal y apretadamente. Flores sésiles, actinomorfas, adpresas, de 7.6-10 mm de largo, de 2.8-5.3 $\mathrm{mm}$ de diámetro; brácteas florales amarillo-anaranjadas, ovadas a anchamente ovadas, de 6-8.8 mm de largo, de 3.4-8.8 mm de ancho, el ápice agudo, más cortas que los sépalos; sépalos anaranjados en la base y amarillos en el ápice, oblongos, asimétricos, de 7.6-11 $\mathrm{mm}$ de largo, de 3.9-6.3 $\mathrm{mm}$ de ancho, los dos externos carinados, el ápice redondeado; pétalos blancos, lanceolados, de 6.2-10 $\mathrm{mm}$ de largo, de 2-4.5 $\mathrm{mm}$ de ancho, el ápice agudo, más cortos que los sépalos; estambres en dos series de diferente longitud; la más corta adnada a la parte basal central del pétalo, los filamentos blancos, cilíndricos, de 3.7-5.4 mm de largo, la serie más larga de 6-6.8 mm de largo; anteras triangulares, amarillas, basifijas, de 1.6-2 $\mathrm{mm}$ de largo en ambas series de estambres; pistilodio verde, de 3.8-6.4 mm de largo, ovoide; estilo ausente; inflorescencia femenina de 20-50 cm de alto, con ca. 14 ramas primarias; pedúnculo verde, cilíndrico, de 47.5-84 cm de largo, de 3.5-11 mm de diámetro; brácteas inferiores del pedúnculo lanceoladas, de 15-34.7 cm de largo, el ápice acuminado, mucho más largas que los entrenudos; brácteas superiores del pedúnculo lanceoladas, de 8-17 cm de largo, el ápice acuminado, más largas que los entrenudos; brácteas primarias ovadas, de 3-4.2(-7.5) $\mathrm{cm}$ de largo, de 1.3-2.6 cm de ancho, el ápice acuminado, las inferiores más largas que el pedículo primario, las superiores más cortas que el pedículo primario; brácteas se- 
Martínez-Correa et al.: Nueva especie de Catopsis (Bromeliaceae) de México

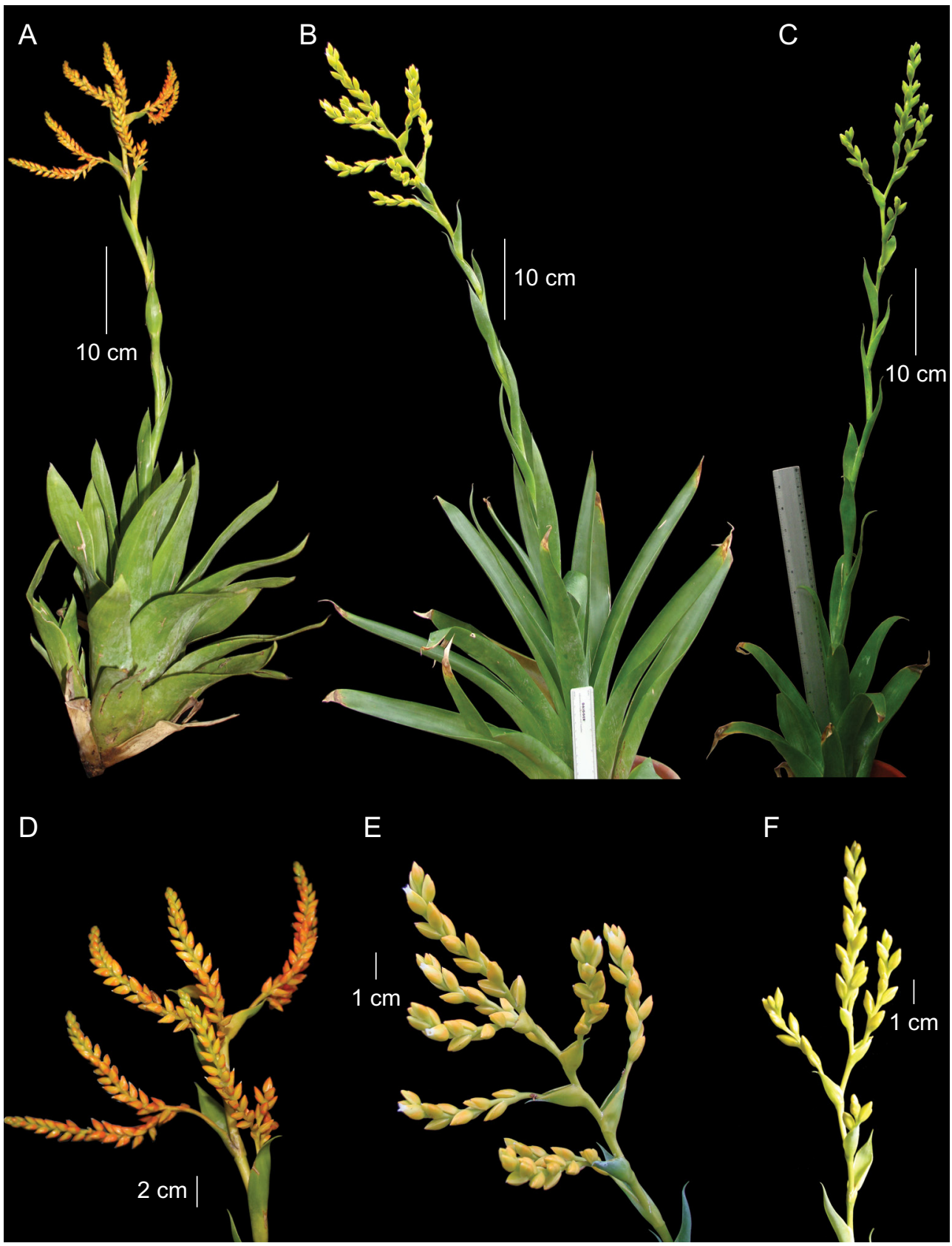

Fig. 5. Catopsis occulta Mart.-Correa, Espejo \& López-Ferr. A. planta masculina; B. planta femenina; D. inflorescencia masculina; E. inflorescencia femenina. Catopsis berteroniana (Schult. \& Schult. f.) Mez; C. planta hermafrodita; F. inflorescencia. 
cundarias lanceoladas, de ca. $8.7 \mathrm{~mm}$ de largo, de ca. $6.6 \mathrm{~mm}$ de ancho, el ápice agudo, más cortas que el pedículo secundario; espigas 8-19, de 8.6-10.6 cm de largo, de 0.9-1.5 $\mathrm{cm}$ de diámetro, con ca. 12-18 flores dispuestas helicoidal y laxamente. Flores sésiles, actinomorfas, ascendentes, de 1.2-1.5 $\mathrm{cm}$ de largo, de 6-8.5 $\mathrm{mm}$ de diámetro; brácteas florales verdes en la base y amarillas en el ápice, ovadas, de 9-11 mm de largo, de 6.8$11 \mathrm{~mm}$ de ancho, el ápice agudo, más cortas que los sépalos; sépalos amarillos, oblongos, asimétricos, de 1.2-1.4 cm de largo, de 6.4-10 mm de ancho, los dos externos con una prominente carina, el ápice redondeado; pétalos blancos, lanceolado-triangulares, de 1-1.5 cm de largo, de 4.2-6.5 mm de ancho, el ápice agudo, iguales o más largos que los sépalos; estaminodios en dos series de diferente longitud, los más cortos adnados a la parte basal central de los pétalos, filamentos blancos, lineares y aplanados, los más largos de 4.7-5.7 mm, los más cortos de 3.3-4.4 mm; ovario verde, ovoide, de 1-1.2 $\mathrm{cm}$ de largo, de 4-4.9 $\mathrm{mm}$ de diámetro, estilo de 1.5-3.9 $\mathrm{mm}$ de largo, estigma del tipo simple erecto (sensu Brown y Gilmartin, 1989). Cápsula parda, ovoide de 1.2-1.8 cm de largo, de 6.3-7.5 mm de diámetro, el ápice agudo. Semillas pardas, fusiformes, de 1.5-2 mm de largo, con un apéndice apical plumoso, enrollado y pardo, de 3.4-4.1 cm de largo y un apéndice basal de 2.1-2.4 mm de largo.

Tipo: MÉXICO. Chiapas, municipio de Ocozocoautla de Espinosa, $14.2 \mathrm{~km}$ después del Carrizal, autopista Malpaso - Tuxtla Gutiérrez, 1649'22" N, 93²2'30" W, 1011 m s.n.m., matorral de leguminosas, 27 julio 2011, N. Martínez-Correa, A. Espejo, A. R. López-Ferrari, J. Ceja y A. Mendoza 232 (holotipo: UAMIZ §).

Paratipos: MÉXICO. Chiapas, municipio de Berriozábal, 12 km antes de Ocozocoautla, viniendo de Mal Paso, 16⒋'11" N, 93²0'56" W, 1030 m s.n.m., bosque tropical caducifolio con Jacquinia, 29 ago 2008, A. Espejo, A. R. López-Ferrari, J. Ceja, A. Mendoza, J. García y L. Terreros 7201 (UAMIZ đ)); Berriozábal - Las Vistas, 4 dic 1949, F. Miranda 5828 (MEXU )); municipio de Chicoasén, $2.01 \mathrm{~km}$ al sur de la colonia Agua Zarca, sobre desvío 3.4 km de la carretera La Nueva - Chicoasén, 17²'22.79" N, 937'30.53" W, 1146 m s.n.m., bosque de Quercus (acahual), 15 sep 2009, M. A. del Barco R. 143 (HEM đ̂); municipio de Ocosingo, en estación Chajul, 150 m s.n.m., sabana, 26 ene 1992, E. Martínez S. y R. Lombera 26113 (CHAP +); municipio de Ocozocoautla de Espinosa, $3 \mathrm{~km}$ north of Ocozocoautla along road to Mal Paso, $900 \mathrm{~m}$ a.s.1., seasonal evergreen forest, 5 nov 1971, D. E. Breedlove 21980 (MEXU, MO $\odot$ ); $31.2 \mathrm{~km}$ después de la presa Malpaso sobre la autopista a Tuxtla Gutiérrez, 16 $6^{\circ} 55^{\prime} 56^{\prime \prime} \mathrm{N}, 93^{\circ} 27^{\prime} 30^{\prime \prime} \mathrm{W}, 878 \mathrm{~m}$ s.n.m., restos de selva mediana perennifolia, 27 jul 2011, N. Martínez-Correa, A. Espejo, A. R. López-Ferrari, J. Ceja y A. Mendoza 
230 (UAMIZ ठ); $14.2 \mathrm{~km}$ después de el Carrizal, sobre la autopista Malpaso - Tuxtla Gutiérrez, 16ㅇํ' $22^{\prime \prime} \mathrm{N}, 93^{\circ} 22^{\prime} 30^{\prime \prime} \mathrm{W}, 1011$ m s.n.m., matorral de leguminosas, 27 julio 2011, N. Martínez-Correa, A. Espejo, A. R. López-Ferrari, J. Ceja y A. Mendoza 231

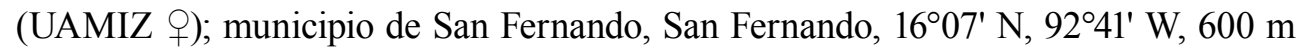
s.n.m., bosque tropical subcaducifolio, 9 sep 2006, H. Gómez D. 1798 (HEM ठ̊); municipio de Tuxtla Gutiérrez, carretera Chiapa de Corzo - Tuxtla Gutiérrez, 16²'00" N, $93^{\circ} 06^{\prime} 00^{\prime \prime} \mathrm{W}, 1050 \mathrm{~m}$ s.n.m., vegetación secundaria derivada de selva baja caducifolia, 18 sep 1991, M. Flores C., A. Espejo y A. R. López-Ferrari 635 (UAMIZ ठ). Oaxaca, Distrito de Juchitán, municipio de Lázaro Cárdenas, $3.6 \mathrm{~km}$ al E de Lázaro Cárdenas, hacia Santa María Chimalapa, entrando por el Mezquite, 16²' N, 9448' W, pinar, 22 ene 1988, R. Torres C. y E. Martínez S. 11233 (CHIP + , MEXU §̋); municipio de Santa María Chimalapa, $3.2 \mathrm{~km}$ adelante de Lázaro Cárdenas, sobre la brecha que parte del Mezquite rumbo a Santa María Chimalapa, 1644'49" N, 9450'55" W, 410 m s.n.m., bosque de pino, 17 nov 1996, J. Ceja, A. Mendoza R., R. Cerros T. y V. Steinmann 386 (UAMIZx2 ô y 9 ); Distrito Mixe, municipio de San Juan Mazatlán, ca. 69 km después de Santiago Tutla rumbo a San Juan Mazatlán, 17²'54" N, 95²6'19" W, 633 m s.n.m., bosque de pino encino, 1 may 2008, A. Espejo, A. R. López-Ferrari, J. Ceja, A. Mendoza, J. García y N. Martínez-Correa 7135 (UAMIZ q); Distrito de Tehuantepec, municipio de Guevea de Humboldt, recorrido al cerro de la Peña Blanca, al SW de la Cumbre, que está a $13.5 \mathrm{~km}$ al N de Guevea, $16^{\circ} 48^{\prime} \mathrm{N}, 95^{\circ} 27^{\prime} \mathrm{W}, 1300 \mathrm{~m}$ s.n.m., bosque mesófilo de montaña, 29 ago 1991, A. Campos V. 3880 (MEXUx2 +); Distrito de Pochutla, municipio de San Pedro Pochutla, $3 \mathrm{~km}$ al N de Chacalapa, 400 m s.n.m., vegetación de sabana con Curatella, Byrsonima y Quercus, 26 sep 1965, J. Rzedowski 21210A (ENCB + , MEXU ô, MICH, TEX). Veracruz, municipio de Soteapan, en Ocotal Chico a $30 \mathrm{~km}$ al NE de Chinameca, bosque de Pinus-Quercus, 3 oct 1981, O. Téllez V., D. Navarro R., P. Arias y G. Pérez 4714 (MEXU ठ)).

Distribución y ecología. Se tienen registros de Catopsis occulta de los estados de Chiapas, Oaxaca y Veracruz en México (Fig. 6). Las poblaciones de la nueva especie crecen entre 150 y 1300 m s.n.m., pero es más frecuente encontrarlas entre 1000 y 1100 m s.n.m. Habitan principalmente en bosques de encino, de pino, de pino-encino, tropicales caducifolios y en matorrales de leguminosas.

Fenología. Florece durante los meses de junio a enero y fructifica de agosto a enero.

Etimología. El epíteto específico hace referencia al hecho de que la especie fue por largo tiempo confundida con C. berteroniana. 
Como mencionamos en párrafos anteriores, el taxon morfológicamente más parecido a Catopsis occulta es C. berteroniana, especie de la cual difiere por las características mostradas en el Cuadro 1 y en las figuras 3 a 5 .

Cuadro 1. Caracteres comparativos entre Catopsis occulta Mart.-Correa, Espejo \& LópezFerr. y C. berteroniana (Schult. \& Schult. f.) Mez.

\begin{tabular}{|c|c|c|}
\hline & Catopsis berteroniana & Catopsis occulta \\
\hline Roseta & $\begin{array}{l}\text { 20-30(35) cm alto x 10-15(20) cm } \\
\text { diámetro }\end{array}$ & $\begin{array}{l}\text { (20)35-50 } \mathrm{cm} \text { alto } \mathrm{x} 20-30 \mathrm{~cm} \\
\text { diámetro }\end{array}$ \\
\hline Inflorescencia & dos veces ramificada & $\begin{array}{l}\text { tres, raramente dos veces } \\
\text { ramificada }\end{array}$ \\
\hline Flores & bisexuales & unisexuales \\
\hline $\begin{array}{l}\text { Brácteas } \\
\text { florales }\end{array}$ & verdes & $\begin{array}{l}\text { amarillo-anaranjadas (masculinas); } \\
\text { verdes en la base y amarillas en el } \\
\text { ápice (femeninas) }\end{array}$ \\
\hline Sépalos & verdes & $\begin{array}{l}\text { anaranjados en la base y amarillos } \\
\text { en el ápice (masculinos); amarillos } \\
\text { (femeninos) }\end{array}$ \\
\hline Pétalos & $\begin{array}{l}\text { lanceolados, iguales o más cortos } \\
\text { que los sépalos }\end{array}$ & $\begin{array}{l}\text { lanceolado-triangulares, iguales o } \\
\text { más largos que los sépalos (en las } \\
\text { flores femeninas) }\end{array}$ \\
\hline $\begin{array}{l}\text { Filamentos de } \\
\text { los estambres }\end{array}$ & laminares & cilíndricos (en flores masculinas) \\
\hline Estilo & ausente & presente, de $1.5-3.9 \mathrm{~mm}$ \\
\hline $\begin{array}{l}\text { Distribución } \\
\text { conocida }\end{array}$ & $\begin{array}{l}\text { México y el Caribe hasta } \\
\text { Sudamérica }\end{array}$ & endémica de México \\
\hline
\end{tabular}

\section{AGRADECIMIENTOS}

Agradecemos a Jacqueline Ceja y Aniceto Mendoza por su invaluable apoyo en el trabajo de campo. A Walter Till por facilitarnos bibliografía valiosa. A los curadores y al personal de los herbarios CHAP, CHAPA, CHIP, CICY, EBT, ECOSUR, ENCB, FCME, HEM, HUMO, IBUG, JBSD, MEXU, MICH, MO, TEX y UAMIZ por otorgarnos todas las facilidades para consultar sus colecciones. A Laura Guglielmone, curadora del herbario TO por ayudarnos con la búsqueda del material tipo de Tillandsia berteroniana. Al Doctorado en Ciencias Biológicas y 


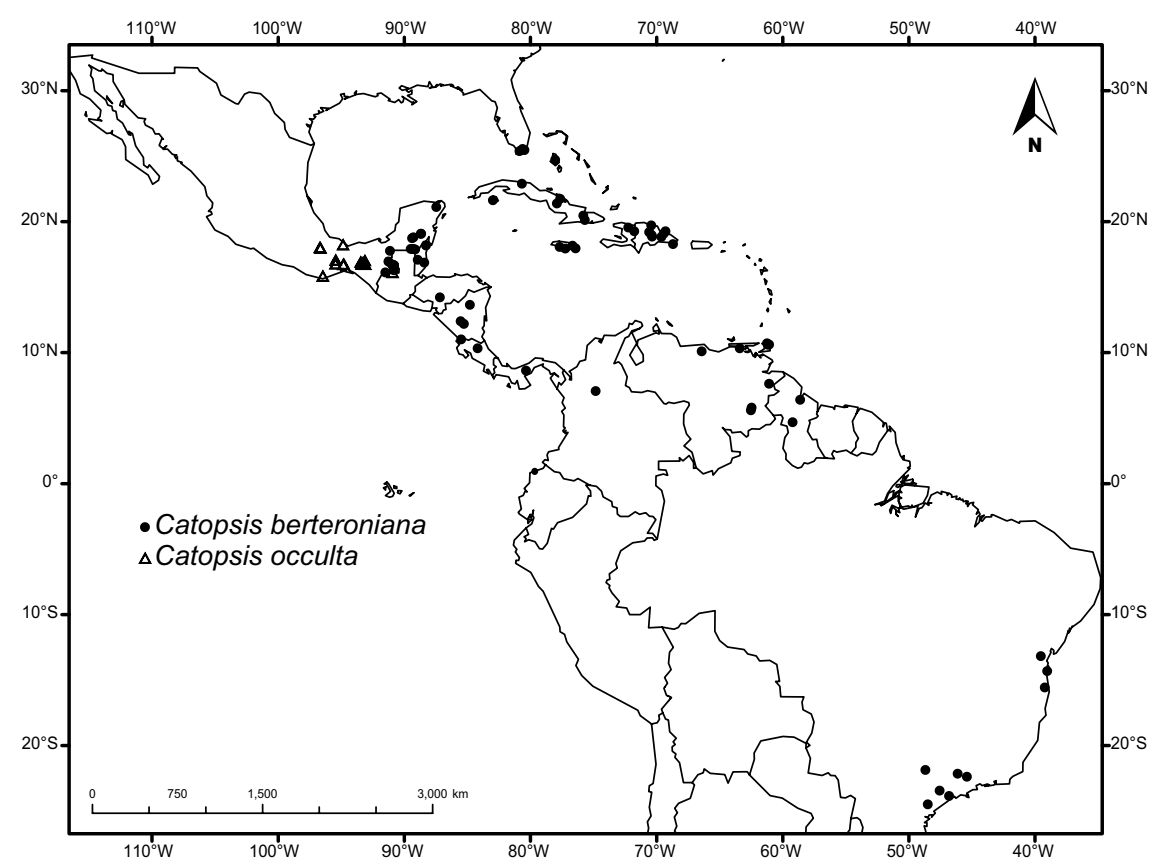

Fig. 6. Distribución geográfica de Catopsis occulta Mart.-Correa, Espejo \& López-Ferr. y C. berteroniana (Schult. \& Schult. f.) Mez.

de la Salud de la Universidad Autónoma Metropolitana, por el patrocinio económico otorgado a la primera autora para realizar una estancia en el Herbario JBSD. Parte de este trabajo se realizó gracias al apoyo otorgado por el Missouri Botanical Garden, a través de la beca Elizabeth E. Bascom, concedida en 2013 a N. Martínez Correa. Los resultados presentados forman parte de la tesis de doctorado de la primera autora y fueron parcialmente financiados por el Consejo Nacional de Ciencia y Tecnología.

\section{LITERATURA CITADA}

Ariza-Julia, L. y A. H. Liogier. 1977. Las bromeliáceas de La Española. Moscosoa 1: 31-38. Barfuss, M., M. R. Samuel, W. Till y T. F. Stuessy. 2005. Phylogenetic relationships in subfamily Tillandsioideae (Bromeliaceae) based on DNA sequence data from seven plastid regions. Amer. J. Bot. 92: 337-351. 
Boggan, J., V. Funk, C. Kelloff, M. Hoff, G. Cremers y C. Feuillet. 1997. Bromeliacae. Checklist of the plants of the Guianas (Guyana, Surinam, French Guiana). 2nd. ed. University of Guyana. Georgetown, Guyana. pp. 67-69.

Brown, G. K. y A. M. Gilmartin. 1989. Stigma types in Bromeliaceae-A systematic survey. Syst. Bot. 14(1): 110-132.

Burt-Utley, K. y J. F. Utley. 1988. New and noteworthy species of Hechtia (Bromeliaceae) from Guerrero, Mexico. Syst. Bot. 13(2): 276-282.

Carabia, J. P. 1941. Las bromeliáceas de Cuba. Mem. Soc. Cubana Hist. Nat. 14(4): 329-347. Espejo Serna, A. 2012. El endemismo en las Liliopsida Mexicanas. Acta Bot. Mex. 100: 195-257. Espejo-Serna, A., A. R. López-Ferrari, I. Ramírez-Morillo, B. K. Holst, H. E. Luther y W. Till. 2004. Checklist of Mexican Bromeliaceae with notes on species distribution and levels of endemism. Selbyana 25: 33-86.

Font Quer, P. 1977. Diccionario de botánica. Ed. Labor. Barcelona, España. 1244 pp.

Forero, E. y A. H. Gentry. 1989. Lista anotada de las plantas del Departamento del Chocó, Colombia. Biblioteca José Jerónimo Triana Núm. 10. Instituto de Ciencias Naturales, Universidad Nacional de Colombia. Bogotá, Colombia. 142 pp.

Gilmartin, A. J. 1965. Las bromeliáceas de Honduras. Ceiba 11: 1-81.

Gómez, M. A. y S. Winkler. 1991. Bromelias en manglares del Pacífico de Guatemala. Rev. Biol. Trop. 39: 207-214.

Grisebach, A. H. R. 1864. Ueber die von Fendler in Venezuela gesammelten Bromeliaceen. Nachr. Königl. Ges. Wiss. Georg-Augusts-Univ. 1: 1-21.

Harms, H. 1930. Bromeliaceae. In: Engler, A. y K. Prantl (eds.). Die natürlichen Pflanzenfamilien, 2a. ed. Vol. 15a. W. Engelmann. Leipzig, Alemania. pp. 65-159.

Harris, J. G. y M. Wolf H. 1997. Plant identification terminology. An illustrated glossary. Spring Lake Publishing. Payson, U.S.A. 197 pp.

Holst, B. K. 1994. Checklist of Venezuelan Bromeliaceae with notes on species distribution by state and levels of endemism. Selbyana 15: 132-149.

Huft, M. J. 1994. Catopsis. Flora Mesoamericana 6: 142-146.

Krömer, T., M. Kessler, B. K. Holst, H. E. Luther, E. J. Gouda, P. L. Ibisch, W. Till y R. Vásquez. 1999. Checklist of Bolivian Bromeliaceae with notes on species distribution and levels of endemism. Selbyana 20: 201-223.

Liogier, H. A. 1974. Bromeliaceae. Flora de Cuba. Instituto Cubano del Libro. Habana, Cuba. pp. 35-37.

Liogier, H. A. y L. F. Martorell. 1982. Bromeliaceae. Flora of Puerto Rico and adjacent islands: A systematic synopsis. Editorial de la Universidad de Puerto Rico. Río Piedras, Puerto Rico. pp. 226-228.

Luther, H. E. 1989. A provisional checklist of the Bromeliaceae of Ecuador. Phytologia 67: 312-330.

Luther, H. E. 1995. An annotated checklist of the Bromeliaceae of Costa Rica. Selbyana 16: 230-234.

Luther, H. E. (comp.) 2012. An alphabetical list of bromeliad binomials. 13 ed. Published by Marie Selby Botanical Gardens \& Bromeliad Society International. Sarasota, USA. $44 \mathrm{pp}$.

Mez, C. 1896. Bromeliaceae. In: de Candolle, C. (ed.). Monogr. Phan. 9: 1-990. 
Moscoso, R. M. 1943. Bromeliaceae. Catalogus florae Dominguensis. Part. I. Spermatophyta. New York, U.S.A. pp. 69-74.

Palací, C. A. 1997. A systematic revision of the genus Catopsis (Bromeliaceae). Ph. D. Dissertation. Department of Botany, University of Wyoming. Laramie, U.S.A. 245 pp.

Proctor, G. R. y R. W. Read. 1972. Bromeliaceae. In: Adams, C. D. Flowering plants of Jamaica (ed.). University of the West Indies. Kona, Jamaica. pp. 41-53.

Rivas, R. M., V. H. Méndez y J. Monge-Nájera. 1997. Distribution of Costa Rican epiphytic bromeliads and the Holdridge life zone system. Rev. Biol. Trop. 45: 1021-1031.

Robinson, H. y D. C. Taylor. 1999. The status of the Pitcairnioideae genera of the Bromeliaceae. Harvard Papers Bot. 4(1): 195-202.

Schultes, J. A. y J. H. Schultes. 1830. Syst. Veg. 7(2): 755-1815.

Smith, L. B. 1938. Bromeliaceae. In: North American Flora 19(2): 61-228.

Smith, L. B. 1943. Bromeliaceae. In: Woodson, R. E. y R. W. Schery (eds.). Contributions toward a flora of Panama. VII. Miscelaneous collections, chiefly by H. von Wedel in Bocas del Toro. Ann. Missouri Bot. Gard. 30: 83-96.

Smith, L. B. y C. R. Lundell. 1940. The Bromeliaceae of the Yucatan Peninsula. Botany of the Mayan Area. Miscellaneous Papers XVI. Carnegie Inst. Wash. Publ. 522: 105-136.

Smith, L. B. y R. J. Downs. 1974. Pitcairnioideae (Bromeliaceae). Flora Neotropica Monograph 14(1): 1-658.

Smith, L. B. y R. J. Downs. 1977. Tillandsioideae (Bromeliaceae). Flora Neotropica Monograph 14(2): 663-1494.

Smith, L. B. \& R. J. Downs. 1979. Bromelioideae (Bromeliaceae). Flora Neotropica Monograph 14(3): 1493-2142.

Smith, L. B. y W. Till. 1998. Bromeliaceae. In: Kubitzki, K. (ed.). The families and genera of vascular plants. IV Monocotyledons. Springer-Verlag. Berlin, Alemania. pp. 74-99.

Standley, P. C. 1928. Bromeliaceae. Flora of the Panama Canal Zone. Contr. U.S. Natl. Herb. 27: 106-109.

Thiers, B. [continuously updated]. Index Herbariorum: A global directory of public herbaria and associated staff. New York Botanical Garden's Virtual Herbarium. http:// sweetgum.nybg.org/ih/

Urban, I. 1903-1911. Bromeliaceae. Flora Portoricensis. Symb. Antill. 4: 138-144.

Vásquez, M. R. 1997. Bromeliaceae. In: Rudas Lleras, A. y Ch. M. Taylor (eds.). Flórula de las reservas biológicas de Iquitos, Perú. Missouri Botanical Garden. St. Louis, USA. 1046 pp.

Versieux, L. M., T. Wendt, R. B. Louzada y M. G. L. Wanderley. 2008. Bromeliaceae da Cadeia do Espinhaço. Megadiversidade 4: 98-110.

Zanoni, T., P. M. Mejía y R. W. Read. 1986. Notas sobre la flora de la isla Española. I. Bromeliaceae. Moscosoa 4: 54-132.

Zavala-Molina, S. V. 2002. Inventario de bromelias epífitas del bosque nebuloso de la montaña El Volcán, El Paraíso, Honduras. Tesis de licenciatura. Escuela Agrícola Panamericana, Zamorano. Zamorano, Honduras. 37 pp. 


\section{APÉNDICE}

Lista de ejemplares revisados.

Catopsis berteroniana (Schult. \& Schult. f.) Mez

Bahamas. Near Nassau, on small trees, 3 feb 1903, A. H. Curtiss 63 (MO). New Providence, on shrubs of rocky flats on each of marsh opposite Teen Scene Camp, along Gladstone Road, 14 mar 1978, D. S. Correll y R. Attrill 49494 (MO). Sin localidad, nov 1890, W. S. Hitchcock s.n. (MO). 16³3-35' N y 88 46'30" W.

Belice, distrito de Corozal, ca. 12 airline $\mathrm{km} \mathrm{W}$ of Little Belize, $18^{\circ} 11^{\prime} \mathrm{N}, 88^{\circ} 17^{\prime} \mathrm{W}, 10$ m a.s.l., tiger savanna, 17 mar 1987, G. Davidse y A. E. Brant 32530 (MEXU, MO). Distrito de Toledo, lower slopes of Peak, Maya Mountains, directly $\mathrm{N}$ of the junction of Richardson Creek and Bladen Branch, 16³3-35' N. 8846'30" W, 300-620 m s.n.m., 4, 6, 8, mar 1987, G. Davidse y A. E. Brant 32109 (MO); Las Sierritas, $20 \mathrm{~km}$ west of Big Creek Settlment. Ridge and western slopes of Cerrito, in the Las Sierritas hills, $16^{\circ} 31^{\prime} 45^{\prime \prime}$ $\mathrm{N}, 88^{\circ} 36^{\prime} 05^{\prime \prime} \mathrm{W}, 160-213$ m s.n.m., vegetation of mixed hardwood species growing on thin soils over exposed limestone, 6 dec 1997, T. Hawkins 1696 (MO); Mile 24 along the

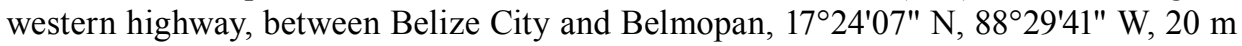
s.n.m., seasonally inundated shrubland, G. Davidse y D. L. Holland 37052 (MO). Sin localidad, 28 ene 1934, C. L. Lundell 1125 (MO).

Brasil, municipio de Olivença, Acuripe, Bahia, 15 ago 1995, G. Hatschbach, M. Hatschbach y J. T. Motta 63334 (MEXU).

Guatemala, departamento Izabal, Livingstone, 9 dic 1988, A. N. Díaz 540 (MO).

Haití, Nord, Massif du Nord, chaine Bonnet Leveque: Morne Bord Choiseul al sur de Milot, $19^{\circ} 35^{\prime} \mathrm{N}, 72^{\circ} 14^{\prime} \mathrm{W}, 700-753 \mathrm{~m}$ s.n.m., vegetación natural y muy rocosa, 24 oct 1985, T. Zanoni 35737 (JBSD).

Honduras, departamento de Gracias a Dios, Tuas: campamento maderero al O de Brus Laguna, $15^{\circ} 46^{\prime} \mathrm{N}, 84^{\circ} 34^{\prime} \mathrm{W}, 0$ m s.n.m., bosque talado, 17-27 abr 1971, C. Nelson y $M$. Hernández 981 (MO).

Jamaica, Trelaway Parish, Burnt Hill between Albert Town and Troy, 560 m s.n.m., forest on jagged exposed limestone hills, 1 feb 1980, A. Gentry y V. Kapos 29313 (MO).

México, Campeche, municipio de Calakmul, a $1.3 \mathrm{~km}$ al SE del poblado Unión 20 de Junio (antes la Moncolona), 1848'29"N, 89¹6'46" W, 209 m s.n.m., selva baja subperennifolia, 21 feb 2003, D. Álvarez, C. Jiménez J. y J. P. Abascal 4126 (MEXU); a 9 km al S de la Nueva Vida, por la carretera Xpujil - Campeche, entrada en el km 25 de ésta, 18 44'3" N, 89²3'41" W, 230 m s.n.m., selva mediana perturbada, con Swietenia macrophylla, 22 nov 1977, E. M. Lira C., E. Martínez, D. Álvarez, S. Ramírez y E. Madrid 331 (MEXUx2); a $2 \mathrm{~km}$ al $\mathrm{N}$ de el Jagüey, camino a Ley de Fomento Agropecuario, 1755'20" N, 89³0'0" W, 250 m s.n.m., selva alta subperennifolia, 15 feb 1998, E. Martínez S., D. Álvarez, B. Sanders y R. Reyna 30134 (MEXU, MO); a 3 km al SE de Pioneros del Río (Mex.), 1752'43" N, 898'30" W, 30 m s.n.m., sabana, 5 ene 1999, E. Martínez S., D. Álvarez M. y S. Ramírez A. $31764 A$ (MEXU); a 9 km al E de Dos 
Apéndice. Continuación.

Naciones, $17^{\circ} 54^{\prime} 52^{\prime \prime} \mathrm{N}, 8^{\circ} 19^{\prime} 8^{\prime \prime} \mathrm{W}, 160$ m s.n.m., selva baja subcaducifolia y matorral de Erythroxylon, Byrsonima, 17 may 1997, E. Martínez S., D. Álvarez y P. Álvaro M. $27240 A$ (MEXU); a 9 km al E de Dos Naciones, 1754'52" N, 89¹9'8" W, 160 m s.n.m., sabana, 23 oct 1997, E. Martínez, D. Álvarez, S. Ramírez, E. Lira y E. Madrid 29256 (MEXU); a $10 \mathrm{~km}$ al SE de Dos Naciones, 1754'16" N, 89¹8'49" W, 170 m s.n.m., selva alta subperennifolia, 23 oct 1997, E. Martínez, D. Álvarez, S. Ramírez, E. Lira y E. Madrid 29311A (MEXU). Chiapas, municipio de La Trinitaria, $5.6 \mathrm{~km}$ después de la desviación a Río Blanco, sobre la carretera Comitán - Palenque, 16²'36" N, 91³0'43" W, 755 m s.n.m., bosque mesófilo de montaña, 29 jul 2011, N. Martínez-Correa, A. Espejo, A. R. López-Ferrari, J. Ceja y A. Mendoza 248 (UAMIZ). Municipio de Las Margaritas, on the western side of Laguna Miramar east of San Quintín, 350 m s.n.m., tropical rain forest Pachira, Cedrela, Ficus, Bursera, 11 feb 1973, D. E. Breedlove 33164 (MO). Municipio de Ocosingo, campamento COFOLASA, a $25 \mathrm{~km}$ del desvío de Frontera Corozal hacia el este sobre la carretera Crucero Corozal - Boca Lacantún, 220 m s.n.m., sabana, 26 may 1987, A. Márquez G., G. Urquijo y E. Martínez 175 (HEM); en ejido Roberto Barrios, a $60 \mathrm{~km}$ al S de Boca Lacantún camino a Chajul, $200 \mathrm{~m}$ s.n.m., selva mediana subcaducifolia, 18 abr 1986, E. Martínez S. 18344 (MEXU); a $5 \mathrm{~km}$ al $\mathrm{S}$ de crucero Corozal, camino a Boca Lacantún, $250 \mathrm{~m}$ s.n.m., selva alta perennifolia, 22 oct 1984, E. Martínez S. y G. Aguilar 8575BIS (MEXUx2, UAMIZ); en crucero Corozal camino Palenque - Boca Lacantún, 180 m s.n.m., selva alta perennifolia, 23 feb 1985, E. Martínez S. 11138 (MEXU); en campamento COFOLASA, a $24 \mathrm{~km}$ al SE de crucero Corozal, camino a Boca Lacantum, 220 m s.n.m., selva media y baja subcaducifolias, 25 feb 1985, E. Martínez S. 11249 (MEXUx2); a 16 km al NW de Boca Lacantum, camino a Palenque, $220 \mathrm{~m}$ s.n.m., selva mediana subcaducifolia, 17 ene 1986, E. Martínez S. 16329 (MEXU); a 4 km al SE de Nuevo Guerrero camino a Boca Lacantún, 560 m s.n.m., sabana, 24 ene 1986, E. Martínez S. 16726 (MEXU); laguna Lacanjá - Chansayab, $30 \mathrm{~km}$ SE del poblado de Lacanjá, 160 m s.n.m., selva alta perennifolia, 25 oct 1985, S. Sinaca C. y G. Ibarra M. 285 (MEXU). Quintana Roo, municipio de Lázaro Cárdenas, población Kantunilkín, área de reserva del CBTA 186, $21^{\circ} 7^{\prime} 0^{\prime \prime} \mathrm{N}, 87^{\circ} 28^{\prime} 0^{\prime \prime} \mathrm{W}, 15 \mathrm{~m}$ s.n.m., selva mediana subperennifolia, 14 nov 2002, $A$. M. Chan D. 134 (CICY). Municipio de Othón P. Blanco, carretera Graciano Sánchez - Nuevo Progreso, unos 15-25 km al oeste de Graciano Sánchez, 194'31" N, 8840'2" W, 0-15 m s.n.m., tintal, 25 oct 1996, G. Carnevali e I. Ramírez 4287 (CICY); 3-4 km al oeste de Margarita Maza, unos $11 \mathrm{~km}$ al oeste de Graciano Sánchez (La Pantera), 194'30" N, 8842'10" W, selva baja inundable (tintales), 2 jul 1997, G. Carnevali, F. May-Pat y M. Gómez J. 4509a (CICY). Tabasco, municipio de Balancán, campamento San Pedro a la orilla del río San Pedro, 10 m s.n.m., vegetación riparia asociada con Rhizophora mangle, 7 dic 1975, P. E. Valdivia Q. 2070 (ENCB).

Nicaragua, departamento Boaco, N slope of Cerro Mombachito and adjacent plain, between Cerro and main road (Boaco-Camoapa), 12²4-25' N, 85³2-33' W, 500-900 m s.n.m., pastures with scattered trees and coffee plantations, 8 oct 1979, W. D. Stevens, B. A. 
Apéndice. Continuación.

Krukoff y A. Grijalva 14745 (MO). Departamento de Chontales, along road from Juigalpa NE toward La Libertad, ca. $17.4 \mathrm{~km}$ NE of Río Mayales, at ford of Río El Bizcocho, $12^{\circ} 12^{\prime} \mathrm{N}, 85^{\circ} 17^{\prime} \mathrm{W}, 350-400 \mathrm{~m}$ s.n.m., pastures gallery forest, $W$. D. Stevens y $B . A$. Krukoff 4110 (MO), ca. $2.3 \mathrm{~km}$ SE of bridge over Quebrada Niscala along road between Acoyapa and Río Oyate, $11^{\circ} 46^{\prime} \mathrm{N}, 8^{\circ} 00^{\prime} \mathrm{W}, 55 \mathrm{~m}$ s.n.m., savanna, 31 ene 1981, W. D. Stevens y B. A. Krukoff 19071 (MO). Departamento Zelaya, Cerro Waylawás (Peñas

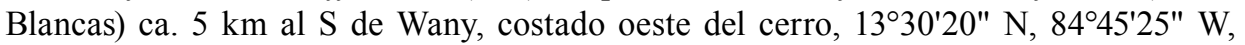
bosque húmedo tropical, 28 oct 1982, A. Grijalva y F. Burgos 1667 (MO); carretera Waslala - Siuna, cerro El Bálsamo, aproximadamente a $30 \mathrm{~km}$ de Waní, 13³9'19" N, 8459'28" W, 360 m s.n.m., abr 1983, F. Ortiz 1070 (MO).

República Dominicana, provincia de El Seibo, Los Haitises, en la bahía de San Lorenzo, en los alrededores de la cueva Arena, parque nacional de Los Haitises, $19^{\circ} 05^{\prime} \mathrm{N}, 69^{\circ} 27^{\prime}$ W, 0-10 m s.n.m., 27 oct 1982, M. Mejía y J. Pimentel 23942 (JBSD). Provincia de la Altagracia, municipio de San Rafael del Yuma, parque nacional del Este, al suroeste de Granchorra, 1.5 hora aprox. en el camino hacia Palmillas y $1.5 \mathrm{~km}$ de la costa, $18^{\circ} 15.5^{\prime}$ N, 68²' W, 10 m s.n.m., 20 ene 1986, B. Peguero, J. Salazar y R. García 278 (JBSD); parque nacional del Este, al sur de Gran Chorra, 1.5 hora caminando a pie por el camino que va a Palmilla, $18^{\circ} 14^{\prime}$ N, 68 $43^{\prime}$ W, 10 m s.n.m., zona pantanosa con Bucida y algunos Conocarpus, 20 ene 1986, R. García, B. Peguero y J. Salazar 896 (JBSD). Provincia de La Vega, $10 \mathrm{NE}$ of Jarabacoa on road to La Vega, 19²1 ' N, 70³5.5' W, 600 m s.n.m., Pinus occidentalis forest, 15 abr 1981, T. Zanoni, M. Mejía y C. Ramírez 12612 (JBSD); Falcombridge Dominicana, mine property, $\mathrm{N}$ of río Yuna, $\mathrm{N}$ of Bonao, on loma el Caribe, $18^{\circ} 59^{\prime} \mathrm{N}, 70^{\circ} 24.5^{\prime} \mathrm{W}, 850 \mathrm{ft}$., Pinus occidentalis forest, $22 \mathrm{abr}$ 1981, T. Zanoni, M. Mejía y C. Ramírez 12862 (JBSD); base de la Cordillera Central, "La Colonia", $0.6 \mathrm{~km}$ de la carretera Duarte (Santo Domingo - Santiago) en el camino a Rincón de Yuboa, $18^{\circ} 52^{\prime} \mathrm{N}$, $70^{\circ} 22^{\prime}$ W, 190 m s.n.m., campos de pastos y cultivos, 3 sep 1982, T. Zanoni, M. Mejía y J. Pimentel 23102 (JBSDx2). Provincia de Monte Plata, margen de Los Haitises, aprox. $7 \mathrm{~km}$ por aire al $\mathrm{N}$ de Bayaguana, cerca de la loma el Mogote (debe ser aprox. arroyo Mermejo, pero los campesinos no conocen este nombre del mapa topográfico), $18^{\circ} 50^{\prime}$ N, 69³8' W, 100 m s.n.m., bosque dentro de praderas rojas, 28 mar 1988, T. Zanoni, J. Pimentel y R. García 40728 (JBSD). Provincia de Puerto Plata, municipio de Sosúa, cordillera septentrional, sección Cabarete, parque nacional El Choco, ubicada en los Mogotes, en la parte norte entrando por el callejón de Doña Tina, $19^{\circ} 43.5^{\prime} \mathrm{N}, 70^{\circ} 28.5^{\prime}$ W, 200-250 m s.n.m., 1 abr 1998, T. Clase, I. de los Ángeles y F. Jiménez 65B (JBSD). Provincia de Samaná, sección Acosta, paraje la Laguna, loma Pilón de Azúcar, 19¹5' N, $422-454$ m s.n.m., matorrales bajos, 3 jun 1996, B. Peguero y A. Veloz 462 (JBSD). Sin provincia indicada, los Haitises, en bahía San Lorenzo, 0 m s.n.m., 2 oct 1976, A. H. Liogier 25468 (JBSD).

Venezuela, estado de Bolívar, carretera fuerte Luepa - Sta. Elena. Parque Nacional Canaima, arbustal, 20 mar 1993, N. Ramírez 3695 (MO); carretera fuerte Luepa - Kanavayen, desvío hacia Riworiwo, Parque Nacional Canaima, arbustal, 24 mar 1993, N. Ramírez 41229 
Apéndice. Continuación.

(MO). Distrito de Piar, Guayaraca, between escarpment and Río Guayaraca, southern base of Auyan-Tepui, 544' N, 62³2' W, 950 m s.n.m., 25-27 nov 1982, G. Davidse y $O$. Huber 22678 (MO). T. F. Delta. Amacuro, departamento de A. Díaz, $60 \mathrm{~km}$ al NE de El Palmar, 8²5' N, 614' W, 120 m s.n.m., 15 mar 1987, G. Aymard C. 5397 (MO). 